\title{
Characteristics and diversity of mutations in regulatory genes of resistance-nodulation-cell division efflux pumps in association with drug-resistant clinical isolates of Acinetobacter baumannii
}

\author{
Bahare Salehi ${ }^{1,2}$, Zohreh Ghalavand $^{2^{*}}$, Abbas Yadegar $^{3^{*}}$ and Gita Eslami ${ }^{2}$
}

\begin{abstract}
Background: This study was aimed to characterize the genetic diversity and expression of three putative resistancenodulation-cell division (RND)-type efflux systems and their contribution to multidrug efflux in clinical isolates of Acinetobacter baumannii.

Methods: Antimicrobial susceptibility testing of 95 A. baumannii isolates was determined by Kirby-Bauer disk diffusion for 18 antibiotics and minimum inhibitory concentration (MIC) of colistin was determined by the broth microdilution method. Moreover, the MIC of five classes of antibiotics was assessed using E-test strips in the presence and absence of phenylalanine-arginine beta-naphthylamide (PABN). Regulatory genes of the RND efflux pumps (adeRS, adeL, adeN and baeSR) were subjected to sequencing. The relative expression of adeB, adeG and adeJ genes was determined by quantitative real-time PCR (qRT-PCR).

Results: Overall, the majority of isolates (94\%) were extensively drug-resistant (XDR). In the phenotypic assay, efflux pump activity was observed in $40 \%$ of the isolates against multiple antibiotics mainly tigecycline. However, we found no efflux activity against imipenem. Several amino acid substitutions were detected in the products of regulatory genes; except in AdeN. Of note, G186V mutation in AdeS was found to be associated with overexpression of its efflux pump. No insertion sequences were detected.

Conclusions: Our findings outlined the role of RND efflux pumps in resistance of A. baumannii to multiple antibiotics particularly tigecycline, and pointed out the importance of a variety of single mutations in the corresponding regulatory systems. Further studies are required to decipher the precise role of RND efflux pumps in multidrug-resistant clinical isolates of A. baumannii.
\end{abstract}

Keywords: Acinetobacter baumannii, RND-type efflux pumps, Efflux pumps inhibitor, Two-component systems

\footnotetext{
*Correspondence: zghalavand@sbmu.ac.ir; a.yadegar@sbmu.ac.ir

2 Department of Microbiology, School of Medicine, Shahid Beheshti University of Medical Sciences, Tehran, Iran

${ }^{3}$ Foodborne and Waterborne Diseases Research Center, Research Institute for Gastroenterology and Liver Diseases, Shahid Beheshti University of Medical Sciences, Tehran, Iran

Full list of author information is available at the end of the article
}

\section{Background}

Acinetobacter baumannii has proved to be one of the highly resilient and adaptable superbugs that have the propensity to cause imminent nosocomial outbreaks worldwide [1]. The isolation of extensively drug-resistant $A$. baumannii (XDR-AB) has drastically limited and nullified most of the therapeutic armamentarium in original author(s) and the source, provide a link to the Creative Commons licence, and indicate if changes were made. The images or other third party material in this article are included in the article's Creative Commons licence, unless indicated otherwise in a credit line to the material. If material is not included in the article's Creative Commons licence and your intended use is not permitted by statutory regulation or exceeds the permitted use, you will need to obtain permission directly from the copyright holder. To view a copy of this licence, visit http://creativecommons.org/licenses/by/4.0/. The Creative Commons Public Domain Dedication waiver (http://creativeco mmons.org/publicdomain/zero/1.0/) applies to the data made available in this article, unless otherwise stated in a credit line to the data. 
healthcare settings [1-3]. Genomic plasticity and genetic variabilities; including mutations in endogenous structural or regulatory genes and insertion of mobile genetic elements, can account for the development of various resistance mechanisms in XDR-AB isolates. Besides, intrinsic resistance determinants such as chromosomally encoded carbapenemase genes, decreased membrane permeability, and efflux systems are inherent in A. baumannii. Of note, in clinical practice, alterations in efflux system regulators, in a single step, can boost efflux activity through overexpression and lead to the extrusion of a wide range of substrates [4-6]. Antibiotic extrusion occurs mainly through overexpression of efflux pumps. In particular, resistance-nodulation-cell division (RND) superfamily pumps are ubiquitous in Gram-negative bacteria and are remarkable for their ability to be selected after exposure to an antibiotic [7].

Overexpression of three chromosomally encoded Acinetobacter drug efflux (Ade) RND systems; AdeABC [8], AdeFGH [9], AdeIJK [10], has been reported in evolving multidrug-resistant (MDR) A. baumannii isolates [6, 11]. These tripartite assemblies of RND efflux pumps span across two membranes, and consist of an inner membrane located RND transport protein with a substratebinding site that pumps drugs out utilizing proton motive force [8-10]. AdeRS two-component regulatory system (TCS) controls AdeABC expression, the LysR-type transcriptional regulator AdeL regulates AdeFGH, and the TetR transcriptional regulator AdeN represses AdeIJK. Point mutations, deletions, or insertions in each of these regulatory systems can affect their respective pump expression $[9,12-16]$.

Although several studies have showed the role of AdeRS in controlling the AdeABC efflux pump, some contradictory results have revealed no correlation between the overexpression of AdeABC pump and AdeRS mutations, elucidating that other mechanisms may be involved [14, 15, 17, 18]. BaeSR is another TCS that has been suggested to regulate AdeABC and likewise AdeIJK [19, 20]. TCSs are signal transduction pathways comprised of a sensor kinase (SK) and its cognate response regulator (RR), which are capable of sensing and mediating drastic and immediate adaptive responses to extracellular and/or intracellular stimuli leading to modulation of gene expression $[21,22]$.

The objective of the current study was to determine the genetic mutations in the cognate regulators of three Ade efflux systems, and their gene expression in XDR and MDR isolates of $A$. baumannii recovered from different clinical specimens at a Hospital in Tehran, Iran.

\section{Methods}

\section{Bacterial isolates and identification}

A total of 95 non-repetitive A. baumannii isolates were collected from inpatients mostly with respiratory tract infections, bacteraemia, and wound infections between August 2016 and February 2017 from an educational hospital in Tehran, Iran. Species identification was performed by the API20NE system (Biomérieux, Marcy-l'Étoile, France), and PCR amplification of the $b l a_{\text {OXA-51-like gene [23] and } r p o B}$ sequencing [24]. This study was approved by the local ethics committee of Shahid Beheshti University of Medical Sciences (IR.SBMU. MSP.REC.1396.22).

\section{Antimicrobial susceptibility testing (AST) and efflux pump activity determination}

Antibiotic susceptibility profile was determined by KirbyBauer disk diffusion for 18 antibiotics (Mast Co., Merseyside, UK) on Mueller-Hinton (MH) agar (Merck, Germany), and minimum inhibitory concentration (MIC) of colistin was determined by broth microdilution method in Mueller-Hinton broth (Merck, Germany), as described by the clinical and laboratory standards institute (CLSI) [25]. Colistin sulfate powder was purchased from Sigma-Aldrich (St. Louis, MO, USA). Escherichia coli ATCC 25922 and Pseudomonas aeruginosa ATCC 27853 strains were used as quality control. Results were interpreted according to the CLSI guidelines [26].

For verification of RND efflux pumps activity, the MIC of agents from five classes of antibiotics including imipenem, levofloxacin, cefepime, tigecycline, and gentamicin were assessed with E-test strips with and without efflux pump inhibitor (EPI) phenylalanine-arginine beta-naphthylamide (PA $\beta N$ ) (Sigma, St. Louis, Mo., USA). PA $\beta N$ was added to $\mathrm{MH}$ agar cooled to $50{ }^{\circ} \mathrm{C}$ at a final concentration of $25 \mu \mathrm{g} / \mathrm{ml}$. Bacterial suspensions equal to a 0.5 McFarland standard were inoculated on $\mathrm{MH}$ agar and E-test strips were overlaid, then plates were incubated for $18 \mathrm{~h}$ at $37{ }^{\circ} \mathrm{C}$. Four-fold or greater reduction of MIC in the presence of EPI was considered significant [27]. Due to lack of tigecycline breakpoints in CLSI for A. baumannii, the US FDA tigecycline susceptibility breakpoints for Enterobacteriaceae (susceptible $\leq 2 \mu \mathrm{g} / \mathrm{ml}$; intermediate $>2$ and $<8 \mu \mathrm{g} / \mathrm{ml}$; resistant $\geq 8 \mu \mathrm{g} / \mathrm{ml}$ ) were used as MIC interpretation criteria. A. baumannii isolates with non-susceptibility to $\geq 1$ agent in $\geq 3$ antimicrobial categories are considered as MDR, and isolates with nonsusceptibility to $\geq 1$ agent in all but $\leq 2$ antimicrobial categories are defined as XDR [28]. 
Primer design and PCR-sequencing of RND-type efflux pump regulatory genes

Screening of RND efflux transporters adeB, adeG and adeJ, and their regulatory genes adeRS, adeL, adeN and baeSR was examined by PCR. Briefly, isolates were propagated overnight in Luria-Bertani (LB) broth (Merck, Germany) at $37{ }^{\circ} \mathrm{C}$ to recover fresh cultures. Genomic DNA was extracted using the High Pure PCR Template Preparation Kit (Roche Co., Germany) according to the manufacturer's instructions. Gene Runner software version 6.0.28 and Primer3 were used to design specific primers for amplification of full-length sequence for each regulatory gene. The primer sets were purchased from Bioneer Co. (Bioneer, Daejeon, South Korea) (Table 1).
DNA sequencing was performed by the conventional Sanger sequencing method using the ABI 3730XL DNA Analyzer (Bioneer, Daejeon, South Korea). All sequences were analyzed using the NCBI BLAST program (https:// blast.ncbi.nlm.nih.gov/Blast.cgi) and multiple-sequence alignment analyses were performed by using ClustalW2 at the European Bioinformatics Institute website (https:// www.ebi.ac.uk/Tools/msa/clustalw2/) and BioEdit software version 7.2.5 [29].

\section{Total RNA isolation and quantitative real-time PCR (qRT-PCR)}

The expression of adeB, adeG and adeJ RND transporter genes was assessed by quantitative real-time RT-PCR.

Table 1 Primers used in this study

\begin{tabular}{|c|c|c|c|}
\hline Target genes & Primer sequence $\left(5^{\prime}-3^{\prime}\right)$ & Amplicon (bp) & References \\
\hline \multicolumn{4}{|c|}{ PCR and sequencing } \\
\hline OXA-51 & $\begin{array}{l}\text { F:TAATGCTTTGATCGGCCTTG } \\
\text { R:TGGATTGCACTTCATCTTGG }\end{array}$ & 353 & {$[23]$} \\
\hline$r p o B$ & $\begin{array}{l}\text { F: GTGATAARATGGCBGGTCGT } \\
\text { R: CGBGCRTGCATYTTGTCRT }\end{array}$ & 543 & {$[24]$} \\
\hline adeB & $\begin{array}{l}\text { F: TTAACGATAGCGTTGTAACC } \\
\text { R:TGAGCAGACAATGGAATAGT }\end{array}$ & 541 & {$[30]$} \\
\hline adeG & $\begin{array}{l}\text { F: TTCATCTAGCCAAGCAGAAG } \\
\text { R: ATGTGGGCTAGCTAACGGC }\end{array}$ & 652 & {$[9]$} \\
\hline ades & $\begin{array}{l}\text { F: ATTGCACCACCAACCGTAAC } \\
\text { R:TAGCTGGATCAAGCCAGATA }\end{array}$ & 463 & {$[30]$} \\
\hline adeR & $\begin{array}{l}\text { F: ATGAGTGTGTAGGGATAATC } \\
\text { R: TACTACAGAAAATAGCGTAAC }\end{array}$ & 961 & This study \\
\hline ades $^{a}$ & $\begin{array}{l}\text { F: ATGTTAATTAATGTGCGTGGC } \\
\text { R: CAGCTTATATGTTAGGTGTCC }\end{array}$ & 1315 & This study \\
\hline ades & $\begin{array}{l}\text { F: ATGAAAAGTAAGTTAGGAATTAGTAAG } \\
\text { R:TTAGTTATTCATAGAAATTTTTATG }\end{array}$ & 1074 & {$[15]$} \\
\hline baesib & $\begin{array}{l}\text { F1: TAAAGATGAAGAAACAATGGAG } \\
\text { R1: ATCACCTAACTCATCATTTCG }\end{array}$ & 1024 & This study \\
\hline baes $2^{b}$ & $\begin{array}{l}\text { F2: CCAGTTTTAGATAAAGAAGATGC } \\
\text { R2: ACCAGATGCGCTAACTCGAC }\end{array}$ & 1014 & This study \\
\hline baeR & $\begin{array}{l}\text { F: CATTAGGTGGTTTACGTTGTG } \\
\text { R: CTGATGTCGCGGTGAGCTG }\end{array}$ & 845 & This study \\
\hline adel & $\begin{array}{l}\text { F: TGGACGGAGCATAAAAGTTTG } \\
\text { R: CAATTTCTATACCATAAGTTAAGG }\end{array}$ & 1246 & This study \\
\hline adeN & $\begin{array}{l}\text { F: AAACATACCAATGACCATCG } \\
\text { R: GTAGCTACTCCATAATAATTG }\end{array}$ & 842 & This study \\
\hline \multicolumn{4}{|c|}{ RT-PCR analysis } \\
\hline$a d e B$ & $\begin{array}{l}\text { F: AACGGACGACCATCTTTGAGTATT } \\
\text { R: CAGTTGTTCCATTTCACGCATT }\end{array}$ & 84 & {$[15]$} \\
\hline adeG & $\begin{array}{l}\text { F: AACTATGCGGTGCTCAAC } \\
\text { R: GGTGAATTACTTGGTGATGC }\end{array}$ & 218 & {$[5]$} \\
\hline adel & $\begin{array}{l}\text { F: AGCTGGTGCTATGGGCGTTA } \\
\text { R: GCCACCCCATGCAATACG }\end{array}$ & 64 & {$[31]$} \\
\hline$r p o B$ & $\begin{array}{l}\text { F: GAGTCTAATGGCGGTGGTTC } \\
\text { R: ATTGCTTCATCTGCTGGTTG }\end{array}$ & 110 & {$[14]$} \\
\hline
\end{tabular}

a Primer sets designed in this study for adeS gene, but could not successfully amplify it

b Since the complete sequence of the baes gene is $1659 \mathrm{bp}$, we designed two overlapping primer pairs for full-length amplification and sequencing of this gene 
For preparation of total RNA, overnight bacterial cultures were subcultured $1: 100$ in fresh $\mathrm{LB}$ broth at $37^{\circ} \mathrm{C}$ to midlog phase (optical density at $600 \mathrm{~nm}$ [OD600]: ca. 0.60.8). After centrifugation at $8000 \mathrm{~g}$ for $5 \mathrm{~min}$, bacterial pellet was resuspended in RNAlater (Sigma-Aldrich Ltd.), vortexed for $20 \mathrm{~s}$ and incubated for $15 \mathrm{~min}$ at room temperature for RNA stabilization. Total RNA was extracted using High Pure RNA isolation kit (Roche, Germany) as the manufacturer's instructions. Extracted RNA samples were treated with DNase I (CinnaGen Co., Iran) to remove any residual DNA contaminations, and frozen at $-80{ }^{\circ} \mathrm{C}$ until used for expression analysis. The concentration and quality of RNA samples were assessed by measuring absorbance at $260 \mathrm{~nm}$ using a spectrophotometer (Denovix, DS-11, USA). Reverse transcription was carried out in a Mastercycler (Eppendorf, Hamburg, Germany) by using TaKaRa PrimeScript TM RT reagent kits (Takara Bio Inc., Kusatsu, Japan) according to the manufacturer's protocol. Negative-control reactions with an equal amount of RNA sample and all the reagents but without reverse transcriptase were included. PCR amplifications were performed in a Rotor-Gene ${ }^{\circledR} \mathrm{Q}$ (Qiagen, Germany) real-time PCR system using BiofACT ${ }^{\mathrm{TM}}$ 2X Real-Time PCR Master Mix (BIOFACT, South Korea). Specific primers used for qRT-PCR are presented in Table 1. The PCR program was as follows: $95^{\circ} \mathrm{C}$ for $15 \mathrm{~min}$, followed by 40 cycles at $95^{\circ} \mathrm{C}$ for $20 \mathrm{~s}, 56{ }^{\circ} \mathrm{C}$ for $30 \mathrm{~s}$, and $72{ }^{\circ} \mathrm{C}$ for $20 \mathrm{~s}$. A melting curve was run at the end of each qPCR run to ensure that there was only one peak and only one product for each primer pair. Relative expression was calculated by the $2^{-\Delta \Delta C T}$ method and the RNA input was normalized against the housekeeping gene $r p o B$. The expression level of each gene was given as the fold change relative to the transcriptional level of each corresponding gene in strain A. baumannii ATCC 19606 (assigned value of 1.0). All reactions were carried out in duplicate and experiments were repeated for three separate times.

\section{Statistical analysis}

Gene expression differences between groups were analyzed using GraphPad Prism software V. 7.04 (GraphPad Software Inc., San Diego, CA), by one-way analysis of variance (ANOVA) followed by Dunnett's post hoc test correction for multiple comparisons and presented as mean \pm standard deviations (SD). Comparison of categorical variables were assessed by Chi-square or Fisher's exact tests using SPSS software for Windows version 22.0 (IBM Corp, Armonk, NY, USA). A $P$ value $<0.05$ was considered statistically significant.

\section{Nucleotide sequence accession numbers}

Nucleotide sequences of the genes examined in this study are deposited in the NCBI database under the following GenBank accession numbers: adeR, MK344139-MK344153; adeS, MK355485-MK355499; adeL, MK318923-MK318937; adeN, MK344124MK344138; baeS, MK344169-MK344183; baeR, MK344154-MK344168.

\section{Results \\ Bacterial isolates, AST pattern and synergistic effect of PABN}

Of 95 isolates that were confirmed as A. baumannii, 76 $(80 \%)$ of them were collected from intensive care unit (ICU), and the majority of samples were originated from respiratory secretions (Table 2).

Referring to AST results (Table 3); 89 (94\%) isolates were considered as XDR, which all were resistant to carbapenems and among them $66(74 \%)$ isolates were non-susceptible to tigecycline. The six remaining isolates were MDR. All isolates were revealed to be susceptible to colistin $(\leq 1 \mu \mathrm{g} / \mathrm{ml})$. In the phenotypic assessment of the efflux-based mechanism of resistance, all of the isolates were grown in the presence of PA $\beta \mathrm{N}(25 \mu \mathrm{g} / \mathrm{ml})$. PA $\beta \mathrm{N}$ decreased the MIC of cefepime (256-128 to 64-32 $\mu \mathrm{g} / \mathrm{ml})$, gentamicin (25696 to $64-24 \mu \mathrm{g} / \mathrm{ml})$ and tigecycline $(16-4$ to $4-1 \mu \mathrm{g} /$ $\mathrm{ml}) \geq$ four-fold in $38(40 \%)$ isolates, and also decreased the MIC of levofloxacin in only one isolate (four-fold) (32 to $8 \mu \mathrm{g} / \mathrm{ml}$ ). All changes in the MIC and AST pattern of the aforementioned antimicrobials after the addition of PA $\beta \mathrm{N}$ are presented in Table 3. Among 38 isolates, some showed fold reduction in MIC of one

Table 2 Demographics and clinical characteristics of patients involved in this study

\begin{tabular}{ll}
\hline Characteristics & No. (\%) \\
\hline Sex & \\
Male & $60(63 \%)$ \\
Female & $35(37 \%)$ \\
Age (year) (mean \pm SD) & $61.34 \pm 16.1$ \\
Total length of hospitalization (days) (mean \pm SD) & $16.8 \pm 12.05$ \\
Site of isolation & \\
Respiratory secretions & $66(69.4 \%)$ \\
Blood & $12(12.6 \%)$ \\
Wound & $9(9.5 \%)$ \\
Urine & $3(3.2 \%)$ \\
CSF & $3(3.2 \%)$ \\
Catheter & $2(2.1 \%)$ \\
Ward of isolation & \\
ICU & $76(80 \%)$ \\
Surgery & $13(13.7 \%)$ \\
Internal & $4(4.2 \%)$ \\
CCU & $2(2.1 \%)$ \\
\hline
\end{tabular}


Table 3 Antimicrobial susceptibility profile of 95 isolates of A. baumannii, and MIC reductions of the antibiotics in exposure to PABN

\begin{tabular}{|c|c|c|c|c|c|c|c|c|c|}
\hline \multirow{2}{*}{$\begin{array}{l}\text { Antimicrobial } \\
\text { agents }\end{array}$} & \multicolumn{3}{|c|}{ Disk diffusion } & \multicolumn{6}{|c|}{ Without PAßN/with PAßN $(25 \mu \mathrm{g} / \mathrm{ml})$} \\
\hline & S no. (\%) & I no. (\%) & R no (\%) & $\begin{array}{l}\text { MIC range }(\mu \mathrm{g} / \\
\mathrm{ml})\end{array}$ & $\mathrm{MIC}_{50}(\mu \mathrm{g} / \mathrm{ml})$ & $\mathrm{MIC}_{90}(\mu \mathrm{g} / \mathrm{ml})$ & S no. (\%) & I no. (\%) & R no. (\%) \\
\hline Imipenem $^{\mathrm{a}}$ & $2(2)$ & $6(6)$ & $87(92)$ & $0.38-32 / 0.38-32$ & $32 / 32$ & $32 / 32$ & $1(1) / 1(1)$ & $0 / 0$ & $94(99) / 94(99)$ \\
\hline Meropenem & $1(1)$ & 0 & $94(99)$ & - & - & - & - & - & - \\
\hline Doripenem & 0 & $1(1)$ & $94(99)$ & - & - & - & - & - & - \\
\hline Cefotaxime & 0 & $1(1)$ & $94(99)$ & - & - & - & - & - & - \\
\hline Ceftazidime & $1(1)$ & 0 & $94(99)$ & - & - & - & - & - & - \\
\hline Cefepime $^{a}$ & $1(1)$ & $7(7)$ & $87(92)$ & $3-256 / 3-256$ & $256 / 96$ & $256 / 256$ & $1(1) / 1(1)$ & $0 / 0$ & $94(99) / 94(99)$ \\
\hline $\begin{array}{l}\text { Ampicillin-Sulbac- } \\
\text { tam }\end{array}$ & $12(13)$ & $22(23)$ & $61(64)$ & - & - & - & - & - & - \\
\hline $\begin{array}{l}\text { Ticarcillin-clavu- } \\
\text { lanic }\end{array}$ & $1(1)$ & 0 & $94(99)$ & - & - & - & - & - & - \\
\hline $\begin{array}{l}\text { Piperacillin-Tazo- } \\
\text { bactam }\end{array}$ & 0 & 0 & $95(100)$ & - & - & - & - & - & - \\
\hline Gentamicin $^{a}$ & $5(5)$ & $16(17)$ & $74(78)$ & $\begin{array}{l}0.75-256 / \mathbf{0 . 3 8}- \\
256\end{array}$ & $96 / 32$ & $256 / 256$ & $4(4) / 4(4)$ & $6(6) / 14(15)$ & $85(90) / 77(81)$ \\
\hline Amikacin & $11(12)$ & $7(7)$ & $77(81)$ & - & - & - & - & - & - \\
\hline Tobramycin & $46(48)$ & $2(2)$ & $47(50)$ & - & - & - & - & - & - \\
\hline Tetracycline & $6(6)$ & $22(23)$ & $67(71)$ & - & - & - & - & - & - \\
\hline Minocycline & $76(80)$ & $12(13)$ & $7(7)$ & - & - & - & - & - & - \\
\hline Tigecycline $^{\mathrm{a}}$ & - & - & - & $\begin{array}{l}0.016-16 / 0.016- \\
12\end{array}$ & $4 / 2$ & $12 / 6$ & $25(26) / 58(61)$ & $31(33) / \mathbf{2 0}(\mathbf{2 1})$ & $39(41) / \mathbf{1 7}(\mathbf{1 8})$ \\
\hline Cotrimoxazole & $1(1)$ & 0 & $94(99)$ & - & - & - & - & - & - \\
\hline Levofloxacin ${ }^{a}$ & $1(1)$ & 0 & 94 (99) & $\begin{array}{l}0.064-32 / 0.064- \\
32\end{array}$ & $32 / 12$ & $32 / 32$ & $2(2) / 2(2)$ & $13(14) / 13(14)$ & $80(84) / 80(84)$ \\
\hline Ciprofloxacin & $1(1)$ & $1(1)$ & $93(98)$ & - & - & - & - & - & - \\
\hline Gatifloxacin & $2(2)$ & 0 & $93(98)$ & - & - & - & - & - & - \\
\hline Colistin $^{b}$ & - & - & - & $0.047-1.0$ & 0.75 & 1.0 & - & - & - \\
\hline
\end{tabular}

Disks concentration: imipenem, $10 \mu \mathrm{g}$; meropenem, $10 \mu \mathrm{g}$; doripenem, $10 \mu \mathrm{g}$; cefotaxime, $30 \mu \mathrm{g}$; ceftazidime, $30 \mu \mathrm{g}$; cefepime, $30 \mu \mathrm{g}$; ampicillin-sulbactam, $10 / 10 \mu \mathrm{g}$; ticarcillin-clavulanic, 75/10 $\mu$; piperacillin-tazobactam, 100/10 $\mu$ g; gentamicin, $10 \mu \mathrm{g}$; amikacin, $30 \mu \mathrm{g}$; tobramycin, $10 \mu \mathrm{g}$; tetracycline, $30 \mu \mathrm{g}$; minocycline, $30 \mu \mathrm{g}$; trimethoprim/sulfamethoxazole, 1.25/23.75 $\mu$; levofloxacin, $5 \mu \mathrm{g}$; ciprofloxacin, $5 \mu \mathrm{g}$; Gatifloxacin, $5 \mu \mathrm{g}$

PA $\beta$, phenylalanine-arginine beta-naphthylamide; MIC, minimum inhibitory concentration; $\mathrm{MIC}_{50}$ and $\mathrm{MIC}_{90}, \mathrm{MIC}$ for $50 \%$ and $90 \%$ of the isolates, respectively. Changes in MIC and AST pattern after addition of PABN, are shown in bold

a MIC of these antibiotics is determined with and without PA $\beta N$ efflux pump inhibitor

b Since disk diffusion is not accepted for colistin AST, its MIC was assessed according to CLSI

antibiotic and some others showed fold reduction in MIC of two or three antibiotics. Among these antibiotics, PA $\beta \mathrm{N}$ had a higher effect on the MIC of tigecycline. However, the MIC of imipenem was not affected in the presence of PAßN (Table 4). Totally, 15 isolates including 13 isolates with $>$ four-fold reduction in MIC of at least one of the over-mentioned antibiotics, and two isolates without any MIC reduction were selected for further sequence and expression analyses.

\section{Sequence analysis}

Efflux pump genes were detected in all of the 95 isolates. Among regulatory genes, we could not amplify adeS using our designed primer pair. Sequence analysis of the regulatory genes revealed several single nucleotide
Table 4 Thirty eight isolates with $\geq$ four-fold reduction in MIC of at least one antibiotic after addition of PAßN $(25 \mu \mathrm{g} / \mathrm{ml})$

\begin{tabular}{llllll}
\hline $\begin{array}{l}\text { Fold } \\
\text { reduction } \\
\text { in MIC }\end{array}$ & \multicolumn{4}{l}{ Antibiotics (no.) } \\
\cline { 2 - 6 } & Imipenem $^{\text {a }}$ & Levofloxacin & \multicolumn{4}{l}{ Cefepime Tigecycline } & Gentamicin \\
\hline 4 & - & 1 & 11 & 18 & 7 \\
5 & - & - & 3 & 3 & 2 \\
6 & - & - & 3 & 2 & 1 \\
7 & - & - & - & - & 1 \\
\hline
\end{tabular}

Among 38 isolates, some showed fold reduction in MIC of one antibiotic and some others showed fold reduction in MIC of two or three antibiotics. Accordingly, the number of isolates for each fold reduction and each antibiotic is the total number

${ }^{a}$ No fold reduction was observed in MIC of imipenem after addition of PABN 
polymorphisms (SNPs) in the adeR, adeS, adeL, adeN, baeS and baeR genes of the selected isolates. In order to compare the amino acid substitutions and polymorphisms, the reference strains $A$. baumannii AYE, ACICU, ATCC 19606 and ATCC 17978 were included in the analysis. AdeR had I120V and A136V, and AdeS had L172P, G186V, F214L, N268H, S280A, Q281D and Y303F polymorphisms as compared to the reference strains. AdeS component had the highest alteration in its amino acid sequence among all 15 isolates. AdeL had only Q262R mutation. All SNPs found in adeN were silent mutations with no changes in amino acid sequences. BaeS had S437T, S471N and P474S mutations, and BaeR revealed to have only S40N amino acid substitutions in some of the isolates. No insertion sequence (IS) was found. All amino acid substitutions are shown in Table 5.

\section{Relative gene expression of RND efflux systems}

The RNA transcript of the major part of tripartite efflux systems (adeB, adeG and adeJ) in the selected isolates were determined by qRT-PCR in relative to that by ATCC 19606 reference strain. Seven, three and two isolates significantly $(P<0.05)$ overexpressed the adeB $(5.09$ to 19.69-fold), adeG (8.75 to 42.51 -fold) and adeJ (2.86 and 5.85 -fold) genes, respectively (Fig. 1).

\section{Discussion}

The impact of multiple resistance mechanisms in the success of A. baumannii as a notorious pathogen has led to confined treatment regimens for this tenacious microorganism [1]. In the present study, the majority of isolates showed an increased rate of resistance to all the first-line antibiotics in comparison to a previous study from Tehran, Iran [32]. Although carbapenems are the mainstay of treatment, all of our XDR isolates were resistant to carbapenems. Since carbapenem-resistant A. baumannii is listed as the "top priority" pathogen in the summit of critical resistant bacteria by WHO, its emergency for research and discovery to explore novel therapeutic options has been recently highlighted [33, 34].

Hitherto, there has been no consensus for the optimal treatment of A. baumannii-associated infections, in particular hospital-acquired pneumonia and bloodstream infections, caused by XDR isolates that are often carbapenem-resistant. Whereas treatment regimen should be made on the case-by-case basis of antimicrobial susceptibility; but considering the importance of early appropriate action, combination therapy is beneficial to target several resistance determinants. Despite dosage limitation due to the colistin nephrotoxicity, this agent is used as a backbone for salvage therapy of the carbapenem-resistant XDR-AB isolates. Colistin-based therapy in combination with sulbactam and tigecycline, in case of susceptibility, responds better than monotherapy. Fortunately, all of our isolates were susceptible to colistin. However, only $13 \%$ and $26 \%$ of the isolates were susceptible to ampicillin-sulbactam and tigecycline, respectively. The inconsistency in outcomes of colistinbased treatment have made it impotent. As an alternative, tigecycline as a drug of last resort for treatment of $\mathrm{XDR}-\mathrm{AB}$ isolates confers a lower cure rate in cases with bloodstream infections due to low serum concentration, and high rates of non-susceptibility in the studies like ours $[2,33,35,36]$. Consequently, approaching an evidence-based therapy is still controversial. Furthermore, it has been alarming that $A$. baumannii can develop drug resistance under the selective pressure both in vitro and through exposure with different antibiotics especially imipenem [5, 18, 37, 38]. Thus, with attention to concerns over rapidly growing number of resistant organisms, some strategies have been brought up to survey novel effective therapeutic trajectories to restore the efficacy of approved antibiotics. For instance, EPIs can hamper efflux activity and have the potential to be used in combination therapy. RND efflux systems have a major role in resistance to multiple categories of antibiotics, and has been verified via inactivation of efflux pump encoding genes [8-10]. Our results were in contrast to another study in which PA $\beta \mathrm{N}$ reduced the MIC of imipenem in $66 \%$ of imipenem-resistant $A$. baumannii isolates [39]. It is suggested that higher concentration of PA $\beta \mathrm{N}$ inhibitor can bypass efflux activity against imipenem. Considering that almost all of our isolates were resistant to imipenem with MIC of $\geq 16 \mu \mathrm{g} / \mathrm{ml}$, resistance determinants such as reduced permeability of the outer membrane or production of carbapenemases could be involved in the carbapenem resistance in addition to efflux systems [33]. In our previous study, we detected OXA-type encoding genes in these isolates [40]. PA $\beta \mathrm{N}$ also affected the gentamicin-resistant phenotype of eight isolates. Moreover, this EPI reduced the MIC of cefepime- and levofloxacinresistant isolates, but had no impact on their susceptibility patterns. Thus, the contribution of other resistance mechanisms can be deduced [33]. To be noted, PA $\beta \mathrm{N}$ remarkably restored tigecycline susceptibility (from 26 to $61 \%$ ); accordingly, active multidrug efflux pumps conferred tigecycline non-susceptibility in our isolates but the type of the pump is not clarified yet [4]. Tigecycline non-susceptibility has been associated with three RND systems as already mentioned [2, 7, 36]. According to previous studies, AdeABC is the predominant pump conferring acquired resistance to a wide range of antibiotics. It is the only RND pump that extrudes aminoglycosides $[8,13,14,37,41]$. Although the role of this pump in carbapenem resistance is controversial, efflux activity was associated with reduced susceptibility to carbapenems 


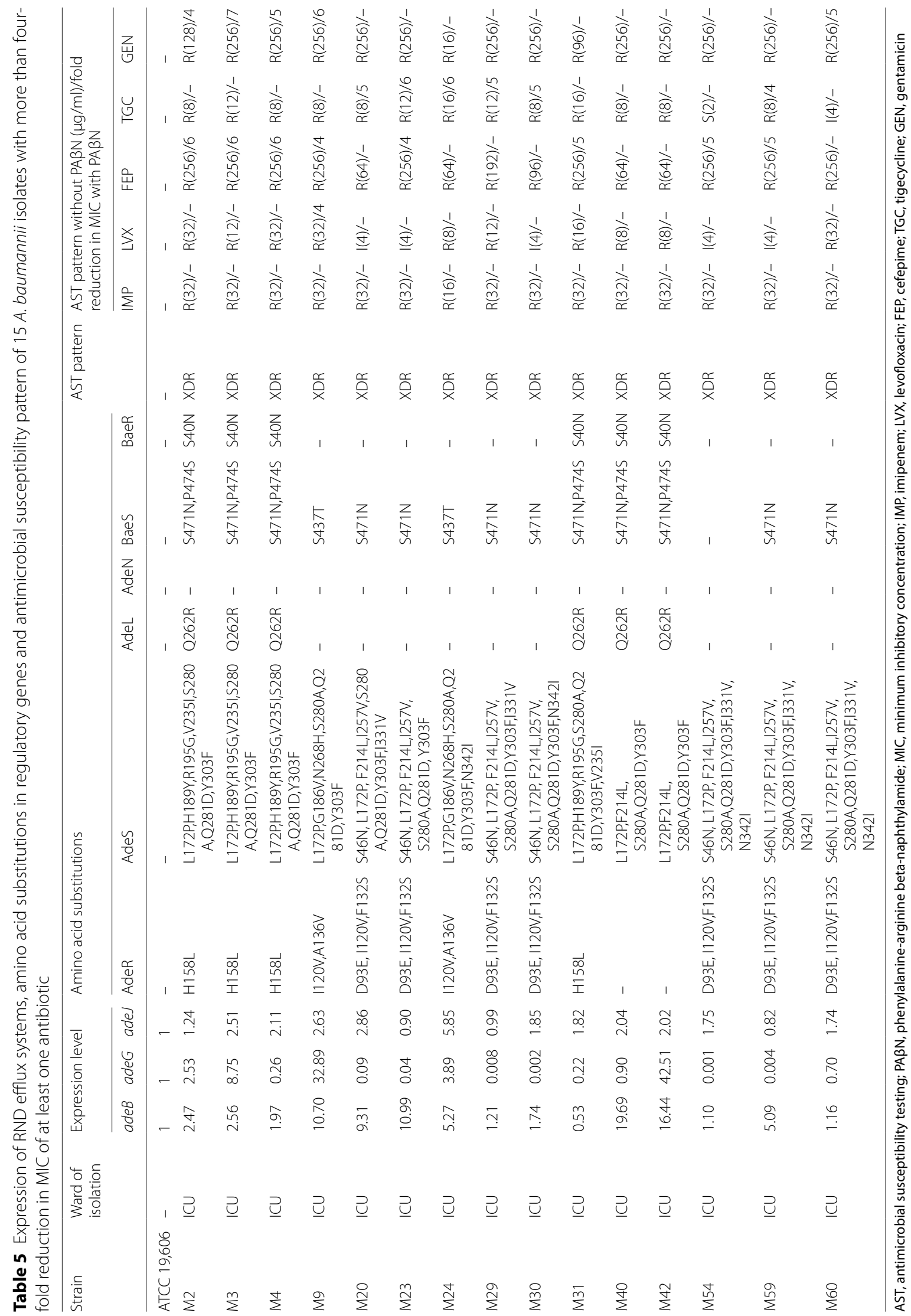


under imipenem-selected stress [5]. AdeRS in the adjacent of AdeABC operon regulates it by transcribing in the opposite direction. Some putative mutations in AdeR are responsible for adeB overexpression: $\mathrm{A} 91 \mathrm{~V}$ and $\mathrm{A} 136 \mathrm{~V}$ in the signal receiver domain [3, 14], D20N in the phosphorylation site [5], and P116L at the first residue of the helix $\alpha 5$ [13]. Among these, A136V polymorphism in the signal receiver of AdeR regulator was detected in two adeB overexpressed isolates (M9 and M24) in our study. In AdeS, which is more prone to mutation, numerous point mutations can boost $a d e B$ expression: G30D located in the periplasmic loop [42], G103D alterations in the histidine kinase, adenylyl cyclase, methyl-accepting chemotaxis protein and phosphatase (HAMP) linker domain [14], G186V in the $\alpha$-helix of the dimerization and histidine phosphotransfer (DHp) domain [3], and T153M in the histidine box [13]. In our study, two isolates (M9 and M24) with overexpression of adeB had G186V mutation, which can alter the conformation of AdeS DHp domain, and then stimulates overexpression of the AdeABC efflux pump [43]. Four of our isolates harbored H189Y located at the C-terminal of the DHp domain of AdeS, which can affect $\mathrm{HK}$ autokinase activity or RR phosphorylation [6]. Furthermore, the coexistence of A136V and G186V polymorphisms respectively in AdeR and AdeS components of two tigecycline-resistant isolates (M9 and M24) is noteworthy. M9 with a 10.70 -fold increase in $a d e B$ expression level showed efflux pump activity for levofloxacin, cefepime and gentamicin antibiotics, and M24 with a 5.27 -fold increase in $a d e B$ expression level showed efflux activity for tigecycline in the phenotypic assay. In line with our results, the coexistence of these two amino acid substitutions have been detected in both tigecyclineresistant and -susceptible isolates; hence, their detailed impact is a matter of controversy $[3,4,6,14]$. The highest expression level of $a d e B$ was detected in M40 (19.69-fold) and M42 (16.44-fold) isolates; two XDR isolates without any reduction in MIC after PA $\beta N$ addition. Additionally, these isolates had a few identical point mutations in each of AdeS, BaeR and BaeS regulators, and had no mutations in AdeR. They harbored none of the renowned mutations and all the detected substitutions were also found in isolates with no pump overexpression. Furthermore, disrupted adeS by ISA balcan lead to tigecycline non-susceptibility and even other antibiotics by enhancing the $\mathrm{Ade} A B C$ overexpression $[6,11,12,41]$. However, this insertion was not detected in our studied isolates.
Regarding the strict regulation of resistance mechanisms under external pressures, the transcriptional regulators were introduced as promising drug targets to overcome resistance. However, the results obtained by Trebosc et al. revealed that there are AdeR-unrelated mechanisms mediating tigecycline resistance, which made AdeR an insufficient target for adjuvant therapy [17]. Tigecycline non-susceptibility can occur as a result of synergistic contribution of AdeIJK with AdeABC [10], while AdeABC has superior influence [18, 38]. The AdeIJK efflux pump is species-specific and contributes to intrinsic resistance to various antibiotics [10]. It is tightly regulated by the product of adeN gene in ca. $800 \mathrm{~kb}$ away from the AdeIJK operon transcribing in the same direction $[16,44]$. Since high-level expression of this pump is toxic for $A$. baumannii, AdeN represses AdeIJK and its disruption diminishes susceptibility following a tolerable expression level. A premature stop codon in the helix $\alpha 9$ sequence at position 211 within the dimerization domain inactivates AdeN [16]. In another study, three types of insertions including ISAba 1 leading to adeN inactivation were detected [11]. Overall, the expression level of adeJ was very slight in our isolates confirming the theory of its lethality for the host. M20 and M24 isolates with minor increases in the expression of adeJ were tigecyclineresistant with pump activity (Table 5). Therefore, the role of other mechanisms in regulation of the AdeIJK operon cannot be ruled out.

The BaeSR is a global regulator and has been associated with tigecycline resistance by controlling AdeIJK and AdeABC pumps. It has been reported that the function of BaeSR occurs through a cross-talk with AdeRS, suggesting the overlap of these two TCS regulons [17, 19-21]. Accordingly, we assessed the BaeSR sequence for any mutation that might be effective in efflux pump expression. In two isolates (M9 and M24) with increased expression level in $a d e B$ and $a d e J$, we found $\mathrm{N} 268 \mathrm{H}$ and S437T polymorphisms in AdeS and BaeS, respectively. These two polymorphisms were not found in the isolates with no increase in the expression of efflux pumps. So far as we know, this is the first investigation on BaeSR and its probable role in the resistance of $A$. baumannii isolates in Iran. A better understanding of the dynamic interaction between AdeRS and BaeSR in the regulation of RND efflux pumps of $A$. baumannii merits further investigations.

(See figure on next page.)

Fig. 1 Relative expression of adeB, adeG, and adeJ genes by qRT-PCR. The relative expression level after being normalized to the expression of the reference gene rpoB were compared relative to that in the reference strain ATCC 19606. The bars represent the means and the error bars the standard errors for the average of the results from independent experiments. Statistical analysis was done by performing one-way ANOVA followed by Dunnett's post hoc test $(P$ value $<0.05)$ 

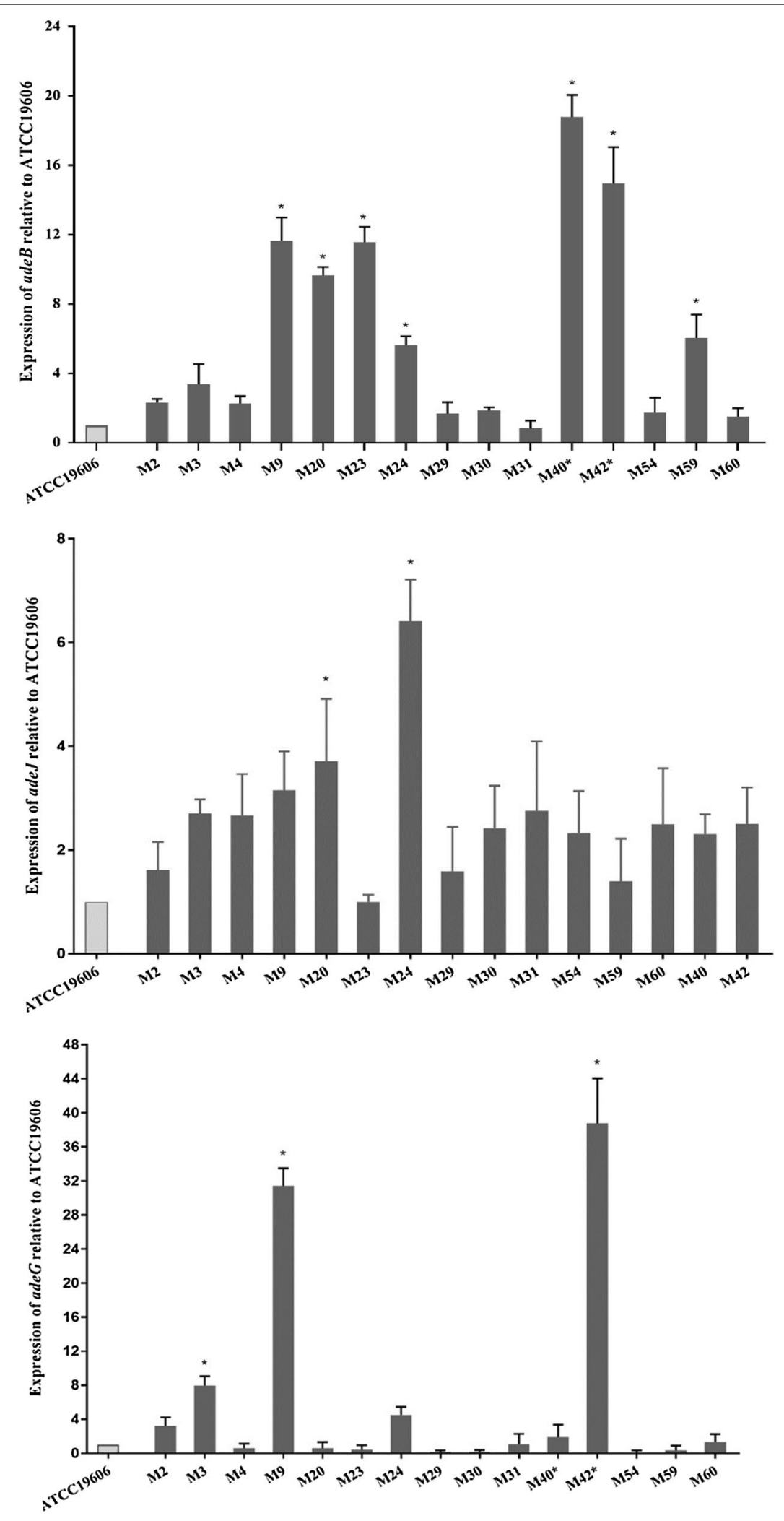
The contribution of AdeFGH to acquired resistance has been proved second to AdeABC. Its overexpression confers decreased susceptibility to several agents. The adeL regulates AdeFGH operon in the upstream of it transcribing in the opposite direction. Deletion of the 11 C-terminal residues, T319K and V139G in the signal recognition domain confer increased expression of ade $G$ [9]. Only Q262R amino acid substitution was found in our isolates. Isolate M42 with the highest expression level (42.51-fold) displayed Q262R substitution in AdeL; but isolate M9 with a 32.89-fold increase in adeG expression showed no alteration. Thus, involvement of other mechanisms is proposed.

The resultant findings of our study elucidated that tigecycline is a substrate for the three aforementioned RND pumps; however, some tigecycline-resistant isolates revealed no pump overexpression. Additional mechanisms contribute to the tigecycline resistance, as previously described $[11,17]$. Among the studied isolates, only isolate M54 was tigecycline-susceptible with no efflux activity and no increase in efflux pump expression, but surprisingly showed a five-fold reduction in the MIC of cefepime indicating efflux activity. Furthermore, two tigecycline-resistant isolates (M29 and M30) with efflux activity for tigecycline and no overexpression of the RND efflux pumps revealed the possibility that other efflux systems play a role in resistance to tigecycline [4].

\section{Conclusions}

To our knowledge, this is the first comprehensive study so far; investigating the gene expression of three prominent RND efflux pumps and the genetic mutations in their regulators among XDR clinical isolates of $A$. baumannii in Iran. Our results revealed that regardless of the contribution of RND-type efflux pumps to resistance against multiple classes of antibiotics especially tigecycline, the increased MIC of antibiotics was not constant as a result of the pump overexpression. Overexpression of efflux pumps supports $A$. baumannii to cope with external stresses and survive in the harsh hospital environments. Evaluation of efflux activity using non-specific EPIs is difficult because of the multiplicity of the pumps. Moreover, the evolution of hypermutator phenotypes and complex sets of resistant determinants have been made it labyrinthine to gain insights into regulatory mechanisms. Consequently, eradication of the newly emerged XDR-AB isolates seems to be strain-dependent. In conclusion, the precise interpretation of discrepant findings obtained with efflux pumps' regulation and expression require (1) characterization of every single mutation in cognate regulators of RND efflux systems, (2) constructing point mutant or its deleted counterpart for pairwise comparison with the parental strain, and (3) development of clinically useful EPIs with an efficient dosage for circumventing efflux pumps. These strategies will be the subject of our future researches, as exerting severe infection control measures and novel therapeutic options to overcome nosocomial XDR-AB strains are desperately needed.

\begin{abstract}
Abbreviations
XDR-AB: Extensively drug-resistant Acinetobacter baumannii; RND: Resistancenodulation-cell division; Ade: Acinetobacter Drug efflux; MDR: Multidrug resistant; TCS: Two-component system; SK: Sensor kinase; RR: Response regulator; AST: Antimicrobial susceptibility testing; MIC: Minimum inhibitory concentration; CLSI: Clinical and laboratory standards institute; EPI: Efflux pump inhibitor; PAßN: Phenylalanine-arginine beta-naphthylamide; PCR: Polymerase chain reaction; qRT-PCR: Quantitative real-time PCR; NCBI: National center for biotechnology information; BLAST: Basic local alignment search tool; CCU: Coronary care unit; ICU: Intensive care unit; CSF: Cerebrospinal fluid: IS: Insertion sequence; IMP: Imipenem; LVX: Levofloxacin; FEP: Cefepime; TGC: Tigecycline; GEN: Gentamicin.
\end{abstract}

\section{Acknowledgments}

The authors are extremely thankful to members of the Department of Microbiology at the School of Medicine, Shahid Beheshti University of Medical Sciences, and also the Foodborne and Waterborne Diseases Research Center, Research Institute for Gastroenterology and Liver Diseases, Shahid Beheshti University of Medical Sciences, Tehran, Iran.

\section{Authors' contributions}

B.S. collected the strains, performed the antimicrobial susceptibility testing and molecular assays, acquisition and interpretation of data, and writing of the manuscript. Z.G. and A.Y. participated in concept and design of the study, and interpretation of data. Z.G., A.Y. and G.E. critically revised the manuscript. All authors have read and approved the final version of the manuscript and the authorship list.

\section{Funding}

The present study was financially supported by Shahid Beheshti University of Medical Sciences, Tehran, Iran (grant number 11247).

Availability of data and materials

All data generated or included during this study are featured in this published article.

\section{Declarations}

Ethics approval and consent to participate Not applicable.

\section{Consent for publication}

Not applicable.

\section{Competing interests}

The authors declare that they have no competing interests.

\section{Author details}

1 Infectious Diseases and Tropical Medicine Research Center, Shahid Beheshti University of Medical Sciences, Tehran, Iran. ${ }^{2}$ Department of Microbiology, School of Medicine, Shahid Beheshti University of Medical Sciences, Tehran, Iran. ${ }^{3}$ Foodborne and Waterborne Diseases Research Center, Research Institute for Gastroenterology and Liver Diseases, Shahid Beheshti University of Medical Sciences, Tehran, Iran.

Received: 4 February 2021 Accepted: 2 March 2021

Published online: 10 March 2021 


\section{References}

1. Peleg AY, Seifert H, Paterson DL. Acinetobacter baumannii: emergence of a successful pathogen. Clin Microbiol Rev. 2008;21:538-82.

2. Peleg AY, Hooper DC. Hospital-acquired infections due to gram-negative bacteria. N Engl J Med. 2010;362:1804-13.

3. Sun JR, Perng CL, Lin JC, Yang YS, Chan MC, Chang TY, et al. AdeRS combination codes differentiate the response to efflux pump inhibitors in tigecycline-resistant isolates of extensively drug-resistant Acinetobacter baumannii. Eur J Clin Microbiol Infect Dis. 2014;33:2141-7.

4. Rumbo C, Gato E, López M, Ruiz de Alegría C, Fernández-Cuenca F, Martínez-Martínez L, et al. Contribution of efflux pumps, porins, and $\beta$-lactamases to multidrug resistance in clinical isolates of Acinetobacter baumannii. Antimicrob Agents Chemother. 2013;57:5247-57.

5. Zhang Y, Li Z, He X, Ding F, Wu W, Luo Y, et al. Overproduction of efflux pumps caused reduced susceptibility to carbapenem under consecutive imipenem-selected stress in Acinetobacter baumannii. Infect Drug Resist. 2018;11:457-67.

6. Yoon EJ, Courvalin P, Grillot-Courvalin C. RND-type efflux pumps in multidrug-resistant clinical isolates of Acinetobacter baumannii: Major role for AdeABC overexpression and aders mutations. Antimicrob Agents Chemother. 2013;57:2989-95.

7. Coyne S, Courvalin P, Périchon B. Efflux-mediated antibiotic resistance in Acinetobacter spp. Antimicrob Agents Chemother. 2011;55:947-53.

8. Magnet S, Courvalin P, Lambert T. Resistance-nodulation-cell divisiontype efflux pump involved in aminoglycoside resistance in Acinetobacter baumannii strain BM4454. Antimicrob Agents Chemother. 2001:45:3375-80.

9. Coyne S, Rosenfeld N, Lambert T, Courvalin P, Perichon B. Overexpression of resistance-nodulation-cell division pump AdeFGH confers multidrug resistance in Acinetobacter baumannii. Antimicrob Agents Chemother. 2010:54:4389-93.

10. Damier-Piolle L, Magnet S, Brémont S, Lambert T, Courvalin P. AdelJK, a resistance-nodulation-cell division pump effluxing multiple antibiotics in Acinetobacter baumannii. Antimicrob Agents Chemother. 2008;52:557-62.

11. Gerson S, Nowak J, Zander E, Ertel J, Wen Y, Krut O, et al. Diversity of mutations in regulatory genes of resistance-nodulationcell division efflux pumps in association with tigecycline resistance in Acinetobacter baumannii. J Antimicrob Chemother. 2018;73:1501-8.

12. Sun JR, Perng CL, Chan MC, Morita Y, Lin JC, Su CM, et al. A truncated AdeS kinase protein generated by ISAba1 insertion correlates with tigecycline resistance in Acinetobacter baumannii. PLoS ONE. 2012;7:e49534

13. Marchand I, Damier-Piolle L, Courvalin P, Lambert T. Expression of the RND-type efflux pump AdeABC in Acinetobacter baumannii is regulated by the AdeRS two-component system. Antimicrob Agents Chemother. 2004;48:3298-304.

14. Hornsey M, Ellington MJ, Doumith M, Thomas CP, Gordon NC, Wareham DW, et al. AdeABC-mediated efflux and tigecycline MICs for epidemic clones of Acinetobacter baumannii. J Antimicrob Chemother. 2010;65:1589-93.

15. Peleg AY, Adams J, Paterson DL. Tigecycline efflux as a mechanism for nonsusceptibility in Acinetobacter baumannii. Antimicrob Agents Chemother. 2007;51:2065-9.

16. Rosenfeld N, Bouchier C, Courvalin P, Perichon B. Expression of the resistance-nodulation-cell division pump AdelJK in Acinetobacter baumannii is regulated by AdeN, a TetR-type regulator. Antimicrob Agents Chemother. 2012;56:2504-10.

17. Trebosc V, Gartenmann S, Royet K, Manfredi P, Tötzl M, Schellhorn B, et al. A novel genome-editing platform for drug-resistant Acinetobacter baumannii reveals an AdeR-unrelated tigecycline resistance mechanism. Antimicrob Agents Chemother. 2016;60:7263-71.

18. Sun JR, Chan MC, Chang TY, Wang WY, Chiueh TS. Overexpression of the adeB gene in clinical isolates of tigecycline-nonsusceptible Acinetobacter baumannii without Insertion mutations in adeRS. Antimicrob Agents Chemother. 2010:54:4934-8.

19. Lin MF, Lin YY, Yeh HW, Lan CY. Role of the BaeSR two-component system in the regulation of Acinetobacter baumannii ade $A B$ genes and its correlation with tigecycline susceptibility. BMC Microbiol. 2014;14:119.

20. Lin MF, Lin YY, Lan CY. The role of the two-component system BaeSR in disposing chemicals through regulating transporter systems in Acinetobacter baumannii. PLoS ONE. 2015;10:e0132843.
21. Kröger C, Kary SC, Schauer K, Cameron ADS. Genetic regulation of virulence and antibiotic resistance in Acinetobacter baumannii. Genes. 2017:8:12.

22. Bhagirath AY, Li Y, Patidar R, Yerex K, Ma X, Kumar A, et al. Two component regulatory systems and antibiotic resistance in Gram-negative pathogens. Int J Mol Sci. 2019;20:1781

23. Turton JF, Woodford N, Glover J, Yarde S, Kaufmann ME, Pitt TL. Identification of Acinetobacter baumannii by detection of the bla OXA-51-like $_{\text {carbap- }}$ enemase gene intrinsic to this species. J Clin Microbiol. 2006:44:2974-6.

24. La Scola B, Gundi VAKB, Khamis A, Raoult D. Sequencing of the rpoB gene and flanking spacers for molecular identification of Acinetobacter species. I Clin Microbiol. 2006;44:827-32

25. Clinical and Laboratory Standards Institute. Methods for dilution antimicrobial susceptibility tests for bacteria that grow aerobically: Eleventh Edition M07. Wayne: CLSI; 2018.

26. Clinical and Laboratory Standard Institute. Performance standard for antimicrobial susceptibility testing: twenty-eighth edition M100. Wayne: CLSI; 2018

27. Pannek S, Higgins PG, Steinke P, Jonas D, Akova M, Bohnert JA, et al. Multidrug efflux inhibition in Acinetobacter baumannii: comparison between 1-(1-naphthylmethyl)-piperazine and phenyl-arginine- $\beta$-naphthylamide. J Antimicrob Chemother. 2006:57:970-4.

28. Magiorakos AP, Srinivasan A, Carey RB, Carmeli Y, Falagas ME, Giske CG, et al. Multidrug-resistant, extensively drug-resistant and pandrugresistant bacteria: an international expert proposal for interim standard definitions for acquired resistance. Clin Microbiol Infect. 2012;18:268-81.

29. Hall TA. BioEdit: a user-friendly biological sequence alignment editor and analysis program for Windows 95/98/NT. Nucleic Acids Symp Ser. 1999:41:95-8

30. Lin $L$, Ling BD, Li XZ. Distribution of the multidrug efflux pump genes, ade $A B C$, adeDE and adelJK, and class 1 integron genes in multiple-antimicrobial-resistant clinical isolates of Acinetobacter baumannii-Acinetobacter calcoaceticus complex. Int J Antimicrob Agents. 2009:33:27-32.

31. Lin MF, Lin YY, Tu CC, Lan CY. Distribution of different efflux pump genes in clinical isolates of multidrug-resistant Acinetobacter baumannii and their correlation with antimicrobial resistance. J Microbiol Immunol Infect. 2017;50:224-31.

32. Lari AR, Ardebili A, Hashemi A. Ader-ades mutations \& overexpression of the AdeABC efflux system in ciprofloxacin-resistant Acinetobacter baumannii clinical isolates. Indian J Med Res. 2018;147:413-21.

33. Viehman JA, Nguyen MH, Doi Y. Treatment options for carbapenem-resistant and extensively drug-resistant Acinetobacter baumannii infections. Drugs. 2014;74:1315-33

34. Tacconelli E, Carrara E, Savoldi A, Harbarth S, Mendelson M, Monnet DL, et al. WHO pathogens priority list working group. Discovery, research, and development of new antibiotics: the WHO priority list of antibioticresistant bacteria and tuberculosis. Lancet Infect Dis. 2017;18:318-27.

35. Kengkla K, Kongpakwattana K, Saokaew S, Apisarnthanarak A, Chaiyakunapruk N. Comparative efficacy and safety of treatment options for MDR and XDR Acinetobacter baumannii infections: a systematic review and network meta-analysis. J Antimicrob Chemother. 2018:73:22-32.

36. Sun Y, Cai Y, Liu X, Bai N, Liang B, Wang R. The emergence of clinical resistance to tigecycline. Int J Antimicrob Agents. 2013:41:110-6.

37. Hornsey M, Loman N, Wareham DW, Ellington MJ, Pallen MJ, Turton JF, et al. Whole-genome comparison of two Acinetobacter baumannii isolates from a single patient, where resistance developed during tigecycline therapy. J Antimicrob Chemother. 2011:66:1499-503.

38. Deng M, Zhu MH, Li JJ, Bi S, Sheng ZK, Hu FS, et al. Molecular epidemiology and mechanisms of tigecycline resistance in clinical isolates of Acinetobacter baumannii from a chinese university hospital. Antimicrob Agents Chemother. 2014;58:297-303.

39. Hou PF, Chen XY, Yan GF, Wang YP, Ying CM. Study of the correlation of imipenem resistance with efflux pumps AdeABC, AdelJK, AdeDE and AbeM in clinical isolates of Acinetobacter baumannii. Chemotherapy. 2012;58:152-8

40. Salehi B, Ghalavand Z, Mohammadzadeh M, Maleki DT, Kodori M, Kadkhoda $\mathrm{H}$. Clonal relatedness and resistance characteristics of OXA-24 and -58 producing carbapenem-resistant Acinetobacter baumannii isolates in Tehran. Iran J Appl Microbiol. 2019;127:1421-9.

41. Ruzin A, Keeney D, Bradford PA. AdeABC multidrug efflux pump is associated with decreased susceptibility to tigecycline in Acinetobacter 
calcoaceticus-Acinetobacter baumannii complex. J Antimicrob Chemother 2007;59:1001-4.

42. Coyne S, Guigon G, Courvalin P, Perichon B. Screening and quantification of the expression of antibiotic resistance genes in Acinetobacter baumannii with a microarray. Antimicrob Agents Chemother. 2010;54:333-40.

43. Sun JR, Jeng WY, Perng CL, Yang YS, Soo PC, Chiang YS, et al. Single amino acid substitution Gly $186 \mathrm{Val}$ in AdeS restores tigecycline susceptibility of Acinetobacter baumannii. J Antimicrob Chemother. 2016;71:1488-92.
44. Yoon EJ, Chabane YN, Goussard S, Snesrud E, Courvalin P, De E, et al. Contribution of resistance-nodulation-cell division efflux systems to antibiotic resistance and biofilm formation in Acinetobacter baumannii. MBio. 2015;6:e00309-e315.

\section{Publisher's Note}

Springer Nature remains neutral with regard to jurisdictional claims in published maps and institutional affiliations.
Ready to submit your research? Choose BMC and benefit from:

- fast, convenient online submission

- thorough peer review by experienced researchers in your field

- rapid publication on acceptance

- support for research data, including large and complex data types

- gold Open Access which fosters wider collaboration and increased citations

- maximum visibility for your research: over $100 \mathrm{M}$ website views per year

At BMC, research is always in progress.

Learn more biomedcentral.com/submissions 